\title{
Cerebral Venous Sinus Thrombosis: A Case Report with Review of Diagnosis and Treatment Strategies
}

\section{Kenton K Murthy ${ }^{1 *}$ and Dalya L Chefitz ${ }^{2}$}

${ }^{1}$ Department of Family Medicine and Community Health, Rutgers Robert Wood Johnson Medical School, USA

${ }^{2}$ Department of Pediatrics, Rutgers Robert Wood Johnson Medical School, USA

\begin{abstract}
Cerebral venous sinus thrombosis (CVST) is an often under-diagnosed, life-threatening condition. We report the case of a 17-year-old female who presented with an acute, severe, on-going, headache. The initial head CT was negative. While the patient's headache initially responded to pain medication and inpatient treatment, the patient subsequently developed left-sided neurological deficits. Subsequent imaging showed thrombosis in the superior sagittal sinus. The patient was treated with Lovenox, as well as thrombolysis using TPA, ballooning and mechanical removal, and soon after her neurological deficits resolved and her headache severity had significantly decreased. The aim of this case report is to provide an example of how early suspicion of CVST, even with initial negative imaging, is critical, as any delay in diagnosis and treatment can cause increased morbidity and mortality.
\end{abstract}

Keywords: Cerebral venous sinus thrombosis; Cerebral venous thrombosis; Headache

Abbreviations: CT: Computed Tomography; CVST: Cerebral Venous Sinus Thrombosis; LMWH: Low Molecular Weight Heparin; MRI: Magnetic Resonance Imaging

\section{Introduction}

Cerebral venous sinus thrombosis (CVST) is an often underdiagnosed condition that can present with a variety of signs and symptoms [1-3]. CVST is a condition in which the venous sinuses become thrombosed, resulting in increased intracranial pressure, toxic edema, seizures and possibly death. While CVST represents just $0.5-1 \%$ of all arterial and venous strokes, it can be a life-threatening disease, especially when there are delays in diagnosis or initiation of therapy due to the variable presentation $[1,2,4-6]$.

The incidence among CVST varies between the adult and pediatric population. Among adults, there are reportedly 3-4 cases per million people per year [6-8]. That number is significantly higher amongst the pediatric group, approaching 7 cases per million people per year $[3,6]$. The most affected group amongst children is the neonatal population, where $40 \%$ of CVST occurs in neonates less than 6 months of age $[3,6,7]$. Some of the adult risk factors for CVST are young, healthy females between the ages of 20-40 [4-6]. Studies show there is a 3:1 ratio of females to males in CVST, which may be due to the use of oral contraceptives, pregnancy and pueripartum, three risk factors that tend to increase thrombosis [1,2,4-6]. Dehydration, infection, and iron-deficiency anemia are risks factors associated with CVST among children $[1,3]$. While risk factors can vary, any medical condition or medication that predisposes a patient to increased clotting can be a cause of CVST $[2,3,6]$. CVST tends to be multifactorial in etiology, with estimates that up to $65 \%$ of patients with CVST had more than 1 risk factor $[2,4]$.

The symptoms of CVST can vary in their presentation, are nonspecific, and as a result, lead to delays in diagnosis [2-4,7]. In young adults, the most common symptom is headache $[4,5,8,9]$. It is estimated that up to $80-90 \%$ of CVST patients first present with either focal, diffuse or migraine-type headaches $[5,6,10]$. Recent studies have suggested that physicians who encounter patients who present with a "thunderclap headache" or "the worst headache of their life" should have CVST high in their differential, in addition to subarachnoid hemorrhage that is often associated with those particular symptoms $[4,5,8,10]$.
Other symptoms are also present and often times may be confused for other more common diseases, such as migraines, meningitis, sinusitis, ischemic or hemorrhagic stroke [2,4-6]. Nearly $70-80 \%$ of all CVSTs are located in the superior sagittal sinus (SSS) $[1,3-6,9,8]$. Signs and symptoms of SSS can include headache, intracranial hypertension and papilledema $[4,5,8]$.

In this case report, we report the symptoms of a young female who presented to the emergency room with an acute headache that had not responded to outpatient, conservative treatment.

\section{Case Report}

A 17-year-old Caucasian female was admitted for onset of severe, on-going headache for the past two days. Past medical history was significant for a 5-year history of chronic migraine headaches, postural orthostatic tachycardia syndrome, Arnold-Chiari Malformation status post craniectomy two years prior to presentation, obesity and anxiety. Patient described the headache as, "the worst headache of her life." Patient stated the headache was in the occipital and frontal areas, and characterized the pain as a stabbing pain associated with nausea and vomiting, which she described was different from her previous migraine headaches. Physical exam revealed normal vital signs, and an alert and oriented female in severe, painful distress. Neurological exam was benign, cranial nerves were intact, Brudzinski and Kernig signs were negative. CT of the head was negative. Patient was admitted for severe, headache refractory to conservative therapy and patient was placed on IV fluids, oxygen, Toradol, Fiorcet, and morphine for pain and Zofran for nausea. Over the course of hospitalization, patient's pain improved from $8 / 10$ severity to $4 / 10$. However, patient would occasionally complain of transient numbness and tingling on the left

*Corresponding author: Kenton K Murthy, Rutgers Robert Wood Johnson Medical School, 1 Robert Wood Johnson Place, Medical Education Bldg 2nd Floor, New Brunswick NJ 08903, USA, Tel: +1 732-445-4636; E-mail: murthykk@rutgers.edu

Received February 18, 2014; Accepted March 21, 2014; Published March 24 2014

Citation: Murthy KK, Chefitz DL (2014) Cerebral Venous Sinus Thrombosis: A Case Report with Review of Diagnosis and Treatment Strategies. J Clin Case Rep 4: 354. doi:10.4172/2165-7920.1000354

Copyright: (c) 2014 Murthy KK, et al. This is an open-access article distributed under the terms of the Creative Commons Attribution License, which permits unrestricted use, distribution, and reproduction in any medium, provided the original author and source are credited. 
side of her face, but no focal weakness or slurred speech was noted and cranial nerves were intact.

Shortly through the hospital course, patient complained of 10/10 headaches, and her nausea and vomiting had resumed. Despite restarting her previous therapy, the patient did not improve. Neurology was consulted, and after evaluation, they stated her headaches were possibly due to pseudotumor cerebri or a worsening of her chronic baseline migraine headaches. However, no papilledema was noted on physical exam.

Soon afterward, the patient complained of left sided numbness, tingling of the face, and left-sided weakness in both the upper and lower extremities. No slurred speech or facial droop were noted on physical exam, and cranial nerves were intact. Neuro-stroke was consulted immediately and they positively identified patient as having true leftsided deficits. CT of the head showed subtle increased density along the superior sagittal and transverse sinuses that were not seen on prior imaging. Subsequent MRV confirmed CT findings and showed new thrombosis of the superior sagittal sinus and the proximal/mid aspect of the right transverse sinuses bilaterally (Figure 1).

A hypercoagulability workup performed which included Factor V, Prothrombin Gene mutation, homocysteine; Protein C\&S, ATIII, ACE, Fibrinogen, Anti-beta 2 glycoprotein, Anti-Cardiolipin, and Lupus screen were all negative. A D-dimer was also performed and was mildly elevated. In addition, TSH, Free T4, Iron, TIBC, and urine pregnancy test were all negative as well. An echocardiogram performed was negative.

In addition to anticoagulation therapy with Lovenox, thrombolysis using TPA, ballooning and mechanical removal of clots was performed to alleviate the neurological symptoms. Subsequent MRV and MRA studies showed improved flow of the cerebral venous sinuses.

The patient reported that her headache severity was $2 / 10$, with improvement from her chronic baseline state. The patient was subsequently discharged from the hospital.

\section{Discussion}

The diagnosis of CVST is often difficult and can lead to a delay in treatment [7]. In fact, the median delay from presentation to diagnosis is 7 days [6]. Therefore, CVST should have a high clinical suspicion in patients who present with the aforementioned risk factors. Imaging and laboratory studies can help in the differentiation between CVST

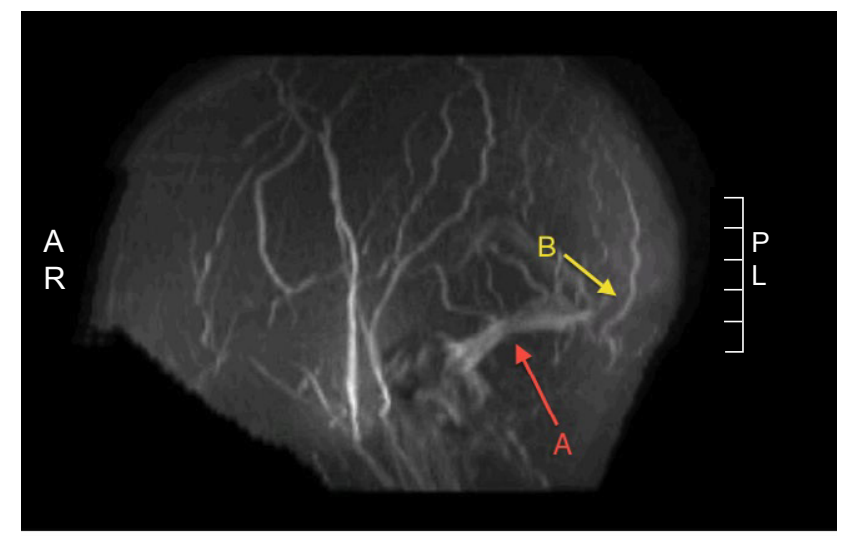

Figure 1: MR Venogram showing the left side of the brain. The " $A$ " arrow shows the left transverse sinus. The arrow marked by "B" shows the cessation of flow to the sagittal sinus and the right transverse sinus. and other diagnoses. The most commonly performed imaging done in the emergency room setting is the head CT $[4,5]$. CT without contrast can reveal findings associated with CVST; however anywhere from 10$40 \%$ of patients with CVST can have a normal head CT $[34,5,9]$. The most common findings on non-contrast head CT are hyperdensity of cortical vein or dural sinuses [4,6,7]. An MRI is more sensitive then a CT for CVST and should be done if there is a high suspicion of CVST and the head CT results were negative $[2,3,5,6]$. A MR Venogram or CT venogram study should also be performed as it can provide the most reliable information $[1,2,4,5]$. A lack of flow in the cerebral veins consists of a positive venogram study.

For laboratory studies, routine blood work such as CBC, chemistry, PTT and PT/INR should be performed to discover any underlying prothrombotic state. A D-Dimer should also be included in any blood work if there is a suspicion of CVST [6]. A negative D-Dimer has a high negative predictive value and thus can safely exclude the diagnosis of CVST; however a positive D-Dimer has a poor predictive positive value and thus does not necessarily confirm a diagnosis of CVST [4]. A lumbar puncture may also provide both diagnostic and therapeutic value $[5,6,10]$. Studies show that CSF abnormalities in CVST are generally nonspecific, but may include a lymphocytic pleocytosis, elevated red blood cell count, and elevated protein $[5,8,10]$.

If CVST is confirmed, then the etiology of the CVST must be discovered. The most common cause of CVST is a prothrombotic condition, and thus studies such as Antithrombin Protein C, Protein S, Factor V Leiden, Prothrombin G20210A mutation, Lupus anticoagulant, anticardiolipin, and anti- $\beta 2$ glycoprotein-I antibodies should also be performed. Particularly in the young adult or pediatric population, the most common causes of CVST are lupus, lymphoma, or leukemia and thus these etiologies should be ruled out [8].

The management of CVST consists of treating the underlying cause of the CVST; however $15 \%$ of patients may not have an obvious cause [3-6,9]. Infectious sources require antibiotic treatment, or brain tumors may require resection to resolve the CVST. The mainstay of treatment for patients with prothrombotic conditions is an anticoagulant such as heparin, enoxaparin, or warfarin $[3,4,5,9]$. Some providers prefer heparin due to the fast reversibility if hemorrhage were to occur while on treatment. Duration of treatment depends on the underlying disorder, but in general, 6-12 months of treatment with coumadin is supported with an INR goal of 2-3 [2-4,6]. Patients with prothrombotic conditions may have to be on anticoagulation for life $[2,7]$.

Recanilzation therapies using fibrinolytics or direct mechanical thrombectomy are another treatment modality [3]. Studies on their effectiveness are small and anecdotal [3,5]. It is recommended that recanalization therapy be used if the patient deteriorates despite adequate anticoagulation, or there is an absolute contraindication to anticoagulation [5]. Aspirin is another treatment that has been used for CVST; however the literature is poor on its effectiveness.

The prognosis of CVST was once very poor; however most studies now show good outcomes. With newer treatment modalities, it is estimated that there is only a $5-10 \%$ mortality rate as a result of CVST [1$4,6,9]$. Studies indicate that there are predictors of increased mortality, namely: depressed consciousness, altered mental status, thrombosis of the deep venous system, right hemisphere hemorrhage, or posterior fossa lesions [3-5,7]. The recurrence risk of CVST is generally quite low; however the studies estimating risk are variable, but in general the recurrence rate is anywhere between $2.8-6.5 \%$ annually with adequate treatment $[5,6,10]$. 
Citation: Murthy KK, Chefitz DL (2014) Cerebral Venous Sinus Thrombosis: A Case Report with Review of Diagnosis and Treatment Strategies. J Clin Case Rep 4: 354. doi:10.4172/2165-7920.1000354

\section{Conclusion}

While CVSTs represents a small fraction of all strokes, a delay in diagnosis can result in death. This report highlights the importance of keeping CVST in the differential diagnosis when treating a young, obese female for ongoing headaches, refractory to treatment. The symptoms of CVST are varied, but those with the most common occlusion in the superior sagittal sinuses often present with signs and symptoms of headache, intracranial hypertension and papilledema. The use of imaging can help aid the diagnosis, with MR or CT venogram being important in detecting the occlusion. The mainstay of treatment for CVST is to resolve the underlying condition, and long-term anticoagulants using LMWH for a minimum of 6 months. Patients with prothrombotic conditions may require life long therapy.

\section{References}

1. Beer-Furlan A, de Almeida CC, Noleto G, Paiva W, Ferreira AA, et al. (2013) Dural sinus and internal jugular vein thrombosis complicating a blunt head injury in a pediatric patient. Childs Nerv Syst 29: 1231-1234.

2. Bousser MG, Ferro JM (2007) Cerebral venous thrombosis: an update. Lancet Neurol 6: 162-170.
3. Dlamini N, Billinghurst L, Kirkham FJ (2010) Cerebral venous sinus (sinovenous) thrombosis in children. Neurosurg Clin N Am 21: 511-527.

4. Bentley JN, Figueroa RE, Vender JR (2009) From presentation to follow-up: diagnosis and treatment of cerebral venous thrombosis. Neurosurg Focus 27: E4.

5. Crassard I, Bousser MG (2004) Cerebral venous thrombosis. J Neuroophthalmol 24: $156-163$

6. Filippidis A, Kapsalaki E, Patramani G, Fountas KN (2009) Cerebral venous sinus thrombosis: review of the demographics, pathophysiology, current diagnosis, and treatment. Neurosurg Focus 27: E3.

7. Okunola PO, Ofovwe GE, Abiodun MT, Azunna CP (2012) Superior sagittal sinus thrombosis complicating typhoid Fever in a teenager. Case Rep Pediatr 2012: 201203.

8. Saposnik G, Barinagarrementeria F, Brown RD Jr, Bushnell CD, Cucchiara B, et al. (2011) Diagnosis and management of cerebral venous thrombosis: a statement for healthcare professionals from the American Heart Association/ American Stroke Association. Stroke 42: 1158-1192.

9. Horowitz MB, Levi El (2005) Neuroendovascular Surgery. (Volume 17), Karger Medical and Scientific Publishers, Basel.

10. De Bruijn SF, Stam J, Kappelle LJ (1996) Thunderclap headache as first symptom of cerebral venous sinus thrombosis. CVST Study Group. Lancet 348: 1623-1625. 1. 